\title{
Effect of Cigarette Smoking and Body Mass Index on Salivary Calcium and Phosphate Concentration in Chronic Periodontitis Patients
}

\author{
Somaye Ansari Moghadam, ${ }^{1}$ Shiva Elmi, ${ }^{2}$ Saide Salimi, ${ }^{3}$ Alireza Ansari Moghaddam, ${ }^{4}$ and Narges \\ Farhad Mollashahi ${ }^{5,}{ }^{*}$ \\ ${ }^{1}$ Department of Periodontology, Oral and Dental Diseases Research Center, Faculty of Dentistry, Zahedan University of Medical Sciences, Zahedan, IR Iran \\ ${ }^{2}$ General Dentist, Zahedan, IR Iran \\ ${ }^{3}$ Genetics of Non-communicable Disease Research Center, Zahedan University of Medical Sciences, Zahedan, IR Iran \\ ${ }^{4}$ Zahedan Health Promotion Research Center, Zahedan University of Medical Sciences, Zahedan, IR Iran \\ ${ }^{5}$ Department of Endodontics, Oral and Dental Diseases Research Center, Faculty of Dentistry, Zahedan University of Medical Sciences, Zahedan, IR Iran \\ "Corresponding author: Narges Farhad Mollashahi, Department of Endodontics, Oral and Dental Diseases Research Center, Faculty of Dentistry, Zahedan University of Medical \\ Sciences, Zahedan, IR Iran. Tel: +98-9153414889. E-mail: nargesfarhadm@gmail.com
}

Received 2015 August 22; Revised 2016 February 11; Accepted 2016 March 14.

\begin{abstract}
Background: Periodontitis is a multi-factorial disease related to the formation of dental plaque. Saliva composition plays a role in plaque formation and in the development of periodontal disease.

Objectives: This study was designed to compare the salivary concentrations of calcium and phosphate between patients with chronic periodontitis (CP) and healthy individuals and to assess the effect of smoking status in this regard.

Patients and Methods: This case control study was conducted on 50 healthy subjects and 56 patients with CP. The two groups were matched in terms of age, sex, and smoking status. After obtaining written informed consents from the subjects, periodontal parameters such as calculus index (CI), plaque index (PI), bleeding index (BI) and clinical attachment loss (CAL) as well as body mass index (BMI) were calculated and recorded. Non-stimulated saliva was collected by the spitting method, and the concentration of calcium and phosphate was measured by spectrophotometry. Data were analyzed using independent t-test. Pearson correlation was utilized to correlate the periodontal parameters with the salivary concentrations of calcium and phosphate in the CP group. Confidence interval and P value were set to $95 \%$ and $\leq 0.05$, respectively.

Results: The mean concentration of phosphate in the saliva of CP patients was significantly higher than that in healthy individuals $(\mathrm{P}=0.008)$. No significant difference was found between the two groups in the mean concentration of calcium in the saliva $(\mathrm{P}=0.145)$. The mean concentrations of calcium and phosphate were not significantly different between non-smoker CP patients and healthy subjects. However, the concentrations of calcium and phosphate in the saliva of smoker CP patients were significantly lower and higher, respectively, than the corresponding values in the healthy smoker group $(\mathrm{P}=0.016$ and $\mathrm{P}=0.037$, respectively). In subjects with $\mathrm{BMI}<25$, the mean concentration of salivary phosphate in $\mathrm{CP}$ patients was significantly higher than that in healthy individuals $(\mathrm{P}=0.005)$. Among the periodontal parameters, only $\mathrm{CI}$ had a significant correlation with the concentration of phosphate in the saliva of patients with $\mathrm{CP}(\mathrm{P}=0.029)$.

Conclusions: Cigarette smoking and BMI are two main confounding factors affecting the correlation of calcium and phosphate concentrations in the saliva and periodontal status.
\end{abstract}

Keywords: Calcium, Phosphate, Saliva, Concentration, Chronic Periodontitis, Smoking

\section{Background}

Periodontitis is a multi-factorial disease associated with the accumulation of plaque and calculus (1). This inflammatory disease is caused by a specific group of microorganisms and is associated with the progressive destruction of bone and the periodontium. Chronic periodontitis (CP) is the most common form of periodontal disease with a higher prevalence in adults (2). The composition of saliva affects the formation of dental plaque and the development of caries and periodontal disease $(2,3)$. The saliva contains proteins and electrolytes such as calcium and phosphate. Studies have shown that several factors, such as age and smoking status, affect the composition of saliva. The concentrations of calcium and phosphate in the saliva significantly increase by aging (2, 4-7). Sah et al. (8). showed a significant increase in the concentration of calcium in the saliva of patients with periodontitis compared with subjects with gingivitis Sewon et al. evaluated the role of smoking in increased concentration of calcium in the saliva and decrease in bone density (9).

The assessment of changes in the concentrations of chemical biomarkers in the saliva can help prevent oral and dental conditions (1). 


\section{Objectives}

This study aimed to assess and compare the concentrations of calcium and phosphate in the saliva of smoker and non-smoker CP patients and healthy individuals.

\section{Patients and Methods}

This study was designed to measure and compare the salivary concentrations of calcium and phosphate in patients with $\mathrm{CP}$ and healthy individuals, and assess the effect of smoking status in this regard. This case-control study was conducted on $56 \mathrm{CP}$ patients and 50 healthy controls (patient companions) presenting to the periodontology department of school of dentistry, Zahedan University of Medical Sciences. The subjects were selected based on the following inclusion and exclusion criteria:

Inclusion criteria:

1) Attachment loss equal or more than $5 \mathrm{~mm}$ in a minimum of 8 teeth,

2) No systemic disease,

3) No history of drug intake in the past one month.

Exclusion criteria:

1) History of periodontal treatment in the past 6 months,

2) Regular use of mouthwash,

3) Chemotherapy and radiotherapy,

4) Pregnancy,

5) Usage of other tobacco products such as hookah, chewing tobacco etc.

The control subjects were selected from patient companions and had no clinical sign or symptom of gingivitis. They had a plaque index $(\mathrm{PI})<15 \%$. All subjects were briefed about the study protocol and objectives, and they signed written informed consent forms voluntarily. The two groups were matched in terms of age, sex, and smoking status. Periodontal examination was conducted using a periodontal probe with $1 \mathrm{~mm}$ accuracy and a periodontal chart including bleeding index (BI), PI, clinical attachment loss (CAL), and calculus index (CI) was completed by a periodontist for patients with $\mathrm{CP}$. For the calculation of body mass index (BMI), the subjects were weighed using a digital scale (Kg) (Beurer GS27 WHD, Germany). Their height was measured using a portable stadiometer $(\mathrm{cm})$ (Seca 213 , UK) while the subjects had no shoes on and were standing tall looking straight ahead.

Data on the general health and smoking status of subjects were collected using a questionnaire. Non-stimulated saliva was collected between 9 a.m. and 11 a.m. using the spitting method. The subjects were requested to refrain from eating, drinking, and tooth brushing 90 minutes prior to sampling. Saliva was collected when the subjects were seated and slightly leaning forward. Saliva was spitted 1 - 2 times per minute for duration of 10 minutes into a sterile test tube (Gamma Counter) (6). After saliva collection, the test tubes were capped, coded, and immediately transferred to a laboratory. Calcium and phosphate concentrations were measured by spectrophotometry using special kits (Zist Chemi). The necessary periodontal treatments were performed for patients with CP after saliva collection. Data were analyzed using an independent sample t-test.

\section{Results}

A total of 106 subjects, including 52 males and 54 females, were evaluated. Among the subjects, 56 had severe $\mathrm{CP}$ and 50 were healthy. For statistical analysis, the patients were divided into two groups of smokers and nonsmokers. Never-smokers and non-smokers were assigned to the non-smoking group, and the remaining subjects were placed in the smoking group. With respect to BMI, the subjects were divided into two groups of $<25$ and $\geq 25$. The distribution of participants in each group was shown in Table 1.

The periodontal parameters, including PI, CAL, CI and $\mathrm{BI}$, in patients with CP are presented in Table 2.

The Pearson correlation coefficient was utilized to find the correlation of periodontal parameters with the salivary concentrations of calcium and phosphate in the CP group (Table 3 ). CI had a significant correlation with the concentration of phosphate in the saliva of patients with $\mathrm{CP}(\mathrm{P}=0.029)$. $\mathrm{CI}$ also had a correlation with salivary concentration of calcium in CP group, but this correlation was not statistically significant $(\mathrm{P}=0.79)$. The correlation of other periodontal parameters with the salivary concentration of calcium and phosphate was not significant.

Comparison of the mean concentration of phosphate and calcium in the saliva of the smoker and non-smoker groups using the independent samples t-test (Table 4) revealed that the mean concentration of calcium and phosphate in the saliva of non-smokers was not significantly different between the two subgroups of healthy individuals and CP patients. However, the mean concentration of phosphate and calcium in the saliva of smokers was significantly different between the two subgroups of healthy individuals and patients with CP. The concentration of calcium in the saliva of smoker CP patients was lower than that in the healthy smoker group, and the concentration of phosphate in the saliva of smoker CP patients was higher than that in the smoker healthy group. In other words, smoking as a confounder affected the correlation between salivary calcium and phosphate concentration and periodontal status (Table 4). 
Table 1. Distribution of Participants in Each Group ${ }^{\mathrm{a}}$

\begin{tabular}{|c|c|c|c|}
\hline Characteristics of Studied Subjects & Severe CP & Healthy & P Value \\
\hline Sex & & & 0.84 \\
\hline Females & $28(50)$ & $26(52)$ & \\
\hline Males & $28(50)$ & $24(48)$ & \\
\hline Smoking status & & & 0.71 \\
\hline Non-smoker & $30(53)$ & $25(50)$ & \\
\hline Smoker & $26(47)$ & $25(50)$ & \\
\hline BMI & & & 0.17 \\
\hline$<25$ & $24(43)$ & $25(50)$ & \\
\hline$\geq 25$ & $32(57)$ & $25(50)$ & \\
\hline
\end{tabular}

${ }^{\mathrm{a}}$ Values are expressed as No. (\%).

Table 2. The Mean of Periodontal Parameters in Patients With CP

\begin{tabular}{lr}
\hline Periodontal Index & Mean \pm SD \\
\hline CAL & $3.96 \pm 2.18$ \\
PI (\%) & $77.78 \pm 18.23$ \\
BI (\%) & $54.54 \pm 9.06$ \\
CI & $2.87 \pm 7.70$ \\
\hline
\end{tabular}

Table 3. The Correlation of Periodontal Parameters With the Salivary Concentrations of Calcium and Phosphate in Patients With CP

\begin{tabular}{|c|c|c|c|c|}
\hline \multirow{2}{*}{$\begin{array}{l}\text { Periodontal } \\
\text { Parameters }\end{array}$} & \multicolumn{2}{|c|}{ Salivary Concentration of Phosphate } & \multicolumn{2}{|c|}{ Salivary Concentration of Calcium } \\
\hline & $\mathbf{P}$ & $\mathbf{R}$ & $\mathbf{P}$ & $\mathbf{R}$ \\
\hline \multirow{2}{*}{ CI } & 0.029 & & 0.79 & \\
\hline & & 0.29 & & 0.036 \\
\hline \multirow{2}{*}{ PI } & 0.244 & & 0.217 & \\
\hline & & 0.15 & & 0.167 \\
\hline \multirow{2}{*}{ BI } & 0.955 & & 0.976 & \\
\hline & & 0.008 & & 0.004 \\
\hline \multirow{2}{*}{ CAL } & 0.978 & & 0.180 & \\
\hline & & 0.004 & & 0.182 \\
\hline
\end{tabular}

Comparison of the mean concentration of calcium and phosphate in subjects with BMI $<25$ showed that the mean concentration of phosphate was higher in the saliva of $\mathrm{CP}$ patients than in that of healthy individuals. However, the mean concentration of calcium in the saliva of the two subgroups of healthy individuals and CP patients was not significantly different. In subjects with $\mathrm{BMI} \geq 25$, the mean concentration of calcium and phosphate in the saliva of the two subgroups of healthy individuals and CP patients was not significantly different. In other words, BMI as a confounder affected the correlation between the salivary concentrations of calcium and phosphate with $\mathrm{CP}$ (Table 4).

\section{Discussion}

The concentrations of calcium and phosphate in the saliva are measured using atomic absorption spectroscopy, inductively coupled plasma-atomic emission, molybdenum-vanadate method, cresolphthalein complexone method and phosphomolybdate ultraviolet (UV) (10-13). In this study, the concentration of calcium and phosphate in the saliva was measured using spectrophotometry. The results showed that the concentration of calcium in the saliva of patients with $\mathrm{CP}$ and healthy individuals was not significantly different, which is in contrast to the results of the studies by Sah et al. (8), Manea et al. (10), and Basima and Omar Husham (11). Manea et al. 
Table 4. The Concentration Mean of Calcium and Phosphate in the Saliva of Studied Groups ${ }^{\mathrm{a}}$

\begin{tabular}{|c|c|c|c|c|}
\hline Study Groups & Salivary Concentration of Phosphate & P Value & Salivary Concentration of Calcium & P Value \\
\hline Healthy & & 0.037 & & 0.016 \\
\hline Smoker & $10.77 \pm 5.69$ & & $5.29 \pm 2.92$ & \\
\hline $\mathrm{CP}$ & $15.65 \pm 9.97$ & & $3.56 \pm 1.94$ & \\
\hline Healthy & & 0.108 & & 0.201 \\
\hline Non-smoker & $10.42 \pm 5.29$ & & $3.52 \pm 0.71$ & \\
\hline $\mathrm{CP}$ & $13.4 \pm 7.73$ & & $4.02 \pm 1.81$ & \\
\hline Healthy & & 0.005 & & 0.427 \\
\hline $\mathrm{BMI}<25$ & $9.09 \pm 3.68$ & & $5.02 \pm 3.1$ & \\
\hline $\mathrm{CP}$ & $13.99 \pm 6.64$ & & $4.38 \pm 1.84$ & \\
\hline Healthy & & 0.095 & & 0.092 \\
\hline $\mathrm{BMI} \geq 25$ & $11.24 \pm 5.97$ & & $4.14 \pm 1.82$ & \\
\hline $\mathrm{CP}$ & $14.78 \pm 10.26$ & & $3.38 \pm 1.8$ & \\
\hline Healthy & & 0.008 & & 0.145 \\
\hline Overall & $10.59 \pm 5.44$ & & $4.4 \pm 2.28$ & \\
\hline $\mathrm{CP}$ & $14.44 \pm 8.83$ & & $3.81 \pm 1.87$ & \\
\hline
\end{tabular}

${ }^{\mathrm{a}}$ Values are expressed as mean $\pm \mathrm{SD}$.

(10) measured the concentration of salivary cations using atomic absorption spectroscopy and found a significant association between the salivary concentration of calcium and periodontitis. Basima et al. (11) showed that the concentration of calcium in the saliva of CP patients was significantly higher than that in healthy individuals. They found that gingival inflammation in patients with $\mathrm{CP}$ increased the gingival crevicular fluid and resulted in an increase in salivary proteins and electrolytes, inconsistent with the findings of our study and those of Kolte et al. (12). In the study by Sah et al. (8), the concentration of calcium in the saliva of patients with periodontitis was higher than that in patients with gingivitis and healthy individuals. The findings of Kolte et al. were in contrast to our results. In their study, the concentration of calcium in the saliva of patients with periodontitis was lower than that in healthy individuals (12). Salivary concentration of calcium in their study was measured using the cresolphthalein complexone method. These controversial results may be due to the different methods used for the measurement of calcium and their accuracy, age of participants, and sample size.

Our study showed that the salivary concentration of phosphate in patients with CP was significantly higher than that in healthy individuals. This result is in accordance with that of Basima et al. and in contrast to that of Kolte et al. (12). Basima et al. (11) used the molybdenum-vanadate method, whereas Kolte et al. measured the salivary concentration of phosphate using the phosphomolybdate UV method.

Cigarette smoking has long been known as a risk factor for periodontal disease. Cigarette smoking can affect the organic and mineral composition of the saliva (2). In our study, in contrast to many others, the number of smoker $\mathrm{CP}$ patients was lower than that of non-smoker CP patients. One reason for this finding is that some of the patients might have hidden their smoking status as the smoking status was determined using a questionnaire and not a diagnostic test for confirmation. Measuring blood nicotine would have added more value to the results, but it was not performed because of the high cost and the need for collecting blood from the subjects.

In the current study, the association between the concentration of calcium and phosphate in the saliva and $\mathrm{CP}$ was evaluated by eliminating the effect of smoking as a confounder. The salivary concentration of calcium was similar between the non-smoker CP patients and the healthy subjects, whereas this value in smoker CP patients was lower than that in healthy smokers. This finding is in contrast to that of Basima et al. (11) but is in agreement with that of Kolte et al. (12). In the study by Basima et al., the concentration of calcium in the saliva of smoker CP patents was higher than that in non-smoker CP patients and nonsmoker healthy individuals. This controversy in the results may be due to the method of classification of smok- 
ers and non-smokers. In our study, the subjects who were not currently smokers were placed in the non-smoking group, but only the never-smokers were assigned to the non-smoking group in the study by Basima et al. Similarly, in the study by Kolte et al., the salivary concentration of calcium in smoker periodontitis patients was higher than that in non-smoker periodontitis patients; this finding reveals that smoking alone can increase the concentration of calcium in the saliva. In the study by Kolte et al. (12), similar to our study, the concentration of calcium in the saliva in the four groups was as follows from the highest to the lowest: smoker CP patients $>$ non-smoker CP patients $>$ healthy smokers $>$ healthy non-smokers. In their study, similar to our study, not smoking at the time of the study was the criterion for assignment of a subject to the non-smoking group, and the subjects who smoked more than 10 cigarettes in the previous years were assigned to the smoking group. Thus, in conjunction with CP, smoking seems to affect the concentration of cations in the saliva.

In the current study, the concentration of phosphate in the saliva of non-smoker CP patients was similar to that in healthy non-smokers. However, the concentration of phosphate in the saliva of smoker patients with CP was higher than that in healthy smokers. This result is in accordance with that of Basima et al. (11) and in contrast to that of Kolte et al. (12).

Obesity has always been a risk factor for periodontal disease, and a correlation has been noted between high BMI and increased risk of periodontitis (13-17). Thus, in the current study, we evaluated the relationship of the concentration of calcium and phosphate in the saliva with BMI. The concentration of calcium and phosphate in the saliva of patients with BMI $\geq 25$ was not significantly different between the CP patients and healthy subjects. The concentration of calcium in the saliva of subjects with BMI $<25$ was also similar in the two subgroups of CP patients and healthy subjects. The concentration of phosphate in the saliva of subjects with $\mathrm{BMI}<25$ was higher in $\mathrm{CP}$ patients than in healthy subjects. However, Basima et al. found that the salivary concentration of minerals was correlated with the nutritional status of individuals.

Among the periodontal parameters in our study, CI was correlated with the concentration of phosphate in the saliva of subjects with CP. This result may be due to the higher concentration of phosphate in the saliva of patients with $\mathrm{CP}$. The increased mineral content of the saliva may enhance the mineralization of dental plaque and accelerate the formation of calculus. In our study, the correlation between the changes in phosphate and calcium concentrations in the saliva and the periodontal parameters (BI, PI, and CAL) was not significant. In the study by Basima et al. (11), PI and calcium concentration in the saliva were corre- lated, and CAL and the concentration of phosphate in the saliva had an inverse correlation in smokers.

Similar studies with a larger sample size are required to better elucidate this subject. Accurate methods such as blood nicotine measurement must be employed in future studies to ensure no tobacco use. Moreover, more precise methods should be used to measure the concentrations of calcium and phosphate in the saliva.

\subsection{Conclusion}

Based on the results of this study, the concentration of calcium was not significantly different in the saliva of $\mathrm{CP}$ patients and healthy individuals, but the concentration of phosphate was higher in the saliva of CP patients. The assessment of the effect of cigarette smoking revealed that the concentration of phosphate was higher and the concentration of calcium was lower in the saliva of smoker $\mathrm{CP}$ patients than in that of healthy smokers. Moreover, $\mathrm{CI}$ was correlated with the concentration of phosphate in the saliva.

\section{Acknowledgments}

We would like to thank to the vice chancellor for research of Medical Sciences University of Zahedan for approving and financially supporting this study.

\section{Footnotes}

Authors' Contribution: Study concept and design: Somaye Ansari Moghadam; gathering of data: Shiva Elmi; analysis and interpretation of data: Alireza Ansari Moghaddam; drafting of the manuscript: Narges Farhad Mollashahi; critical revision of the manuscript for important intellectual content: Somaye Ansari Moghadam; statistical analysis: Alireza Ansari Moghaddam; administrative, technical, and material support; study supervision: Somaye Ansari Moghadam.

Funding/Support: Zahedan University of Medical Sciences.

\section{References}

1. Page RC. Milestones in periodontal research and the remaining critical issues. J Periodontal Res. 1999;34(7):331-9. [PubMed: 10685357].

2. Zappacosta B, Persichilli S, Mordente A, Minucci A, Lazzaro D, Meucci $\mathrm{E}$, et al. Inhibition of salivary enzymes by cigarette smoke and the protective role of glutathione. Hum Exp Toxicol. 2002;21(1):7-11. [PubMed: 12046726].

3. Macgregor ID, Edgar WM. Calcium and phosphate concentrations and precipitate formation in whole saliva from smokers and nonsmokers. J Periodontal Res. 1986;21(4):429-33. [PubMed: 2942670]. 
4. Banderas-Tarabay JA, Zacarias-D Oleire IG, Gardu-o-Estrada R, AcevesLuna E, Gonzalez-Begne M. Electrophoretic analysis of whole saliva and prevalence of dental caries. A study in Mexican dental students. Arch Med Res. 2002;33(5):499-505. doi: 10.1016/S0188-4409(02)003958 .

5. Sevon L, Laine MA, Karjalainen S, Doroguinskaia A, Helenius H, Kiss E, et al. Effect of age on flow-rate, protein and electrolyte composition of stimulated whole saliva in healthy, non-smoking women. Open Dent J. 2008;2:89-92. doi: 10.2174/1874210600802010089. [PubMed: 19088888].

6. Agha-Hosseini F, Mirzaii-Dizgah I, Moghaddam PP, Akrad ZT. Stimulated whole salivary flow rate and composition in menopausal women with oral dryness feeling. Oral Dis. 2007;13(3):320-3. doi: 10.1111/j.1601-0825.2006.01288.x. [PubMed:17448216].

7. Agha-Hosseini F, Mirzaii-Dizgah I, Mansourian A, Zabihi-Akhtechi G. Serum and stimulated whole saliva parathyroid hormone in menopausal women with oral dry feeling. Oral Surg Oral Med Oral Pathol Oral Radiol Endod. 2009;107(6):806-10. doi: 10.1016/j.tripleo.2009.01.024. [PubMed: 19386515].

8. Sah N, More SP, Bhutani H. Estimation and comparison of salivary calcium levels in healthy subjects and patients with gingivitis and periodontitis: Cross-sectional biochemical study. Arch Oral Sci Res. 2012;2(1):13-6.

9. Sewon L, Laine M, Karjalainen S, Doroguinskaia A, LehtonenVeromaa M. Salivary calcium reflects skeletal bone density of heavy smokers. Arch Oral Biol. 2004;49(5):355-8. doi: 10.1016/j.archoralbio.2003.11.004. [PubMed: 15041482].

10. Manea A, Nechifor M. Research on plasma and saliva levels of some bivalent cations in patients with chronic periodontitis (salivary cations in chronic periodontitis). Rev Med Chir Soc Med Nat Iasi. 2014;118(2):439-49. [PubMed: 25076713].

11. Basima GA, Omar Husham A. Detection of salivary flow rate and minerals in smokers and non smokers with chronic periodontitis (Clinical and Biochemical study).J Baghdad College Dentistry. 2012;24(1):687.

12. Kolte AP, Kolte RA, Laddha RK. Effect of smoking on salivary composition and periodontal status. J Indian Soc Periodontol. 2012;16(3):350-3. doi: 10.4103/0972-124X.100909. [PubMed: 23162327].

13. Al-Zahrani MS, Bissada NF, Borawskit EA. Obesity and periodontal disease in young, middle-aged, and older adults. J Periodontol. 2003;74(5):610-5. doi: 10.1902/jop.2003.74.5.610. [PubMed: 12816292].

14. Nishida N, Tanaka M, Hayashi N, Nagata H, Takeshita T, Nakayama $\mathrm{K}$, et al. Determination of smoking and obesity as periodontitis risks using the classification and regression tree method. I Periodontol. 2005;76(6):923-8. doi: 10.1902/jop.2005.76.6.923. [PubMed: 15948686].

15. Nihlani T, Soni S, Patel P. , Prabu D. , Kulkarni S. . Relationship between body mass index and periodontal disease status in vegetarian and mixed diet subjects of udaipur, rajasthan, india. J Orafacial Res. 2011;1(1):11-4.

16. Pataro AL, Costa FO, Cortelli SC, Cortelli JR, Abreu MH, Costa JE. Association between severity of body mass index and periodontal condition in women. Clin Oral Investig. 2012;16(3):727-34. doi: 10.1007/s00784011-0554-7. [PubMed: 21556849].

17. Ekuni D, Mizutani S, Kojima A, Tomofuji T, Irie K, Azuma T, et al. Relationship between increases in BMI and changes in periodontal status: a prospective cohort study.JClin Periodontol. 2014;41(8):772-8. doi: 10.1111/jcpe.12273. [PubMed: 24813869]. 